\title{
Comparison of gut microbiota and allergic reactions in BALB/c mice fed different cultivars of rice
}

\author{
Kei Sonoyama ${ }^{1}$, Toru Ogasawara ${ }^{2}$, Haruka Goto $^{2}$, Tomoyo Yoshida ${ }^{2}$, Naoki Takemura ${ }^{2}$, Reiko Fujiwara ${ }^{2}$, \\ Jun Watanabe ${ }^{3}$, Hiroyuki Ito $^{4}$, Tatsuya Morita ${ }^{4}$, Yoshinari Tokunaga ${ }^{5}$ and Tetsuji Yanagihara ${ }^{6}$ \\ ${ }^{1}$ Research Faculty of Agriculture, Hokkaido University, Sapporo, Hokkaido 060-8589, Japan \\ ${ }^{2}$ Graduate School of Life Science, Hokkaido University, Sapporo, Hokkaido 060-8589, Japan \\ ${ }^{3}$ Creative Research Initiative 'Sousei', Hokkaido University, Sapporo, Hokkaido 001-0021, Japan \\ ${ }^{4}$ Department of Applied Biological Chemistry, Faculty of Agriculture, Shizuoka University, Shizuoka 422-8529, Japan \\ ${ }^{5}$ Chinobeikokuten Co., Ltd, Sapporo, Hokkaido 065-0032, Japan \\ ${ }^{6}$ Hokkaido Prefectural Kamikawa Agricultural Experiment Station, Pippu, Hokkaido 078-0397, Japan
}

(Received 3 April 2009 - Revised 13 July 2009 - Accepted 13 July 2009 - First published online 22 September 2009)

Our preliminary clinical trial showed that consumption of cooked rice of a Japanese common cultivar Yukihikari improved atopic dermatitis associated with a suspected rice allergy, although the underlying mechanisms remain unclear. We hypothesised that the ameliorating effect of Yukihikari on atopic dermatitis is associated with the gut microbiota. BALB/c mice were fed a synthetic diet supplemented with uncooked and polished white rice powder prepared from one of four different cultivars: Yukihikari, rice A (common rice), rice B (brewery rice) and rice C (waxy rice). Denaturing gradient gel electrophoresis of PCR-amplified 16S rRNA gene fragments showed that the composition of faecal microbiota was different between mice fed Yukihikari and those fed rice A. Analysis of the 16S rRNA clone library and species-specific realtime PCR showed that the abundance of Akkermansia muciniphila, a mucin degrader, tended to be lower in mice fed Yukihikari. The incidence of allergic diarrhoea induced by oral administration of ovalbumin in systemically immunised mice was lower in mice fed Yukihikari, albeit with no difference in serum antibodies specific to ovalbumin. In a separate experiment, serum antibody levels specific to orally administered ovalbumin were lower in mice fed Yukihikari. Additionally, the translocation of horseradish peroxidase in isolated segments of ileum and colon tended to be lower in mice fed Yukihikari, suggesting a reduction in gut permeability in mice fed Yukihikari. These data indicate that changes in the gut microbiota of mice fed Yukihikari could be advantageous in the prevention of food allergy.

Rice: Allergy: Gut microbiota: Mice

Rice seed is a cereal consumed in large quantities around the world and is a staple foodstuff in most countries in South and East Asia. So far, several clinical studies have shown that IgEmediated allergy against rice was associated with a subset of patients with atopic dermatitis (AD) in Japan ${ }^{(1-3)}$. Additionally, since Shibasaki et al. ${ }^{(4)}$ first described that a high degree of allergenicity was found in a globulin fraction of rice seed proteins ${ }^{(4)}$, many studies have reported on rice allergenic proteins ${ }^{(5-9)}$. Although avoidance of the intake of allergens is generally one of the emphasised therapeutic suggestions for allergy, it is impractical to strictly remove rice and its products from Asian diets, including that of Japan. Therefore, attempts have been made to eliminate allergenic rice proteins by protease treatment ${ }^{(10)}$, high-pressure treatment $^{(11)}$ and antisense transgene expression ${ }^{(12)}$.

We previously performed a small-scale prospective open clinical trial to examine the effects of consuming Yukihikari, a common rice cultivar developed by the Hokkaido
Central Agricultural Experiment Station in Japan, on AD patients with a suspected rice allergy. A total of thirtyeight patients with a mean age of 5.09 (range 0-38) years were enrolled in the trial and fed Yukihikari as their staple food for 4 weeks. The clinical skin score was improved in twenty-six patients $(68.4 \%)$, unchanged in eleven patients $(29.0 \%)$ and exacerbated in one patient $(2.6 \%)$ after consuming Yukihikari ( $\mathrm{T}$ Yanagihara, unpublished results). However, the contents of the salt-soluble fraction of rice proteins in Yukihikari did not differ from those in other rice cultivars, and the binding capacities of these proteins in Yukihikari to $\mathrm{IgE}$ antibodies in the sera of $\mathrm{AD}$ patients with a suspected rice allergy were also comparable with other cultivars ( $\mathrm{T}$ Yanagihara, unpublished results). Therefore, not only rigorously controlled clinical trials, but also mechanistic studies are needed to clarify the ameliorating effect of Yukihikari on AD associated with rice allergy.

Abbreviations: AD, atopic dermatitis; DGGE, denaturing gradient gel electrophoresis; HRP, horseradish peroxidase; OVA, ovalbumin; rice A, common rice; rice B,

brewery rice; rice $\mathrm{C}$, waxy rice.

* Corresponding author: Dr Kei Sonoyama, fax +81 11706 2496, email ksnym@chem.agr.hokudai.ac.jp 
The commensal microbiota in the intestinal tract plays an important role in the normal development of the immune system $^{(13,14)}$. Therefore, strategies to manipulate the microbiota have been explored in the prevention of the onset of immune diseases such as allergy. Indeed, several clinical interventions showed that administration of probiotic bacterial strains, such as Lactobacillus rhamnosus GG, was beneficial in both the prevention ${ }^{(15)}$ and treatment ${ }^{(16)}$ of early allergic diseases. Additionally, prebiotics, such as indigestible oligosaccharides, have also been shown to promote immune health by selectively stimulating the growth and/or activity of beneficial bacteria, such as bifidobacteria and lactobacilli, in the intestinal tract ${ }^{(17-20)}$. A mixture of long-chain fructooligosaccharide and short-chain galacto-oligosaccharide reportedly reduced the incidence of $\mathrm{AD}$ in formula-fed highrisk infants ${ }^{(21,22)}$. Our animal studies demonstrated that dietary raffinose and $\alpha$-linked galacto-oligosaccharide reduced allergic airway inflammation in ovalbumin (OVA)-sensitised Brown Norway rats ${ }^{(23,24)}$. We also showed that dietary short-chain fructo-oligosaccharide reduced 2,4-dinitrofluorobenzene-induced contact hypersensitivity in BALB/c mice $^{(25)}$. In addition, Fujitani et al. showed that dietary fructo-oligosaccharide reduced infiltration of inflammatory cells and oedema formation in duodenal mucosa using an OVA-induced food allergy model of NC/jic mice ${ }^{(26)}$. Furthermore, Vos et al. reported that consumption of a mixture of long-chain fructo-oligosaccharide and short-chain galactooligosaccharide suppressed allergic airway inflammation in an OVA-induced allergic asthma model of BALB/c mice ${ }^{(27)}$. Therefore, it is postulated that modulation of gut microbiota by some indigestible constituents, for example, resistant starch, in rice seed may be involved in the AD-reducing effect of Yukihikari by counteracting immune dysfunction associated with allergic disease.

Starch digestibility is largely dependent upon the amylose:amylopectin ratio ${ }^{(28)}$. Our previous studies demonstrated that consumption of high-amylose maize starch increased intestinal mucin contents, possibly due to increased production of SCFA, the fermentation products of resistant starch, in the caecum of rats ${ }^{(29,30)}$. This effect was accompanied by reduction of D-galactosamine-induced liver injury ${ }^{(29)}$ and trinitrobenzene sulfonic acid-induced colitis ${ }^{(30)}$ as a result of reduced permeability in the intestinal mucosa. Additionally, Toden et al. ${ }^{(31,32)}$ reported that dietary high-amylose maize starch attenuated dietary protein-induced colonic DNA damage via protecting mucus barrier thinning in rats ${ }^{(31,32)}$. These findings suggest that dietary resistant starch potentiates intestinal mucus barrier function. Furthermore, considering the growing evidence indicating that probiotics such as lactobacilli and bifidobacteria counteract the intestinal mucosal barrier dysfunction associated with allergic disease ${ }^{(33)}$, modulation of gut microbiota by consuming Yukihikari may potentiate the intestinal mucosal barrier. Therefore, an alternative possibility is that some indigestible constituents such as resistant starch may reduce intestinal permeation of food antigens and then prevent food allergy, which may contribute to the AD-reducing effect of Yukihikari.

In different rice cultivars, the amylose content is higher in brewery rices, lower in waxy rices and intermediate in common rices. Therefore, the present study compared Yukihikari with a representative cultivar of common rice (rice A), brewery rice (rice B) and waxy rice (rice C). Additionally, given that prevention of allergy by gut microbiota modulation is independent of the type of allergens, the effect of supplementation with Yukihikari would not be limited to rice allergy. Therefore, the present study investigated gut microbiota composition and OVA-induced allergic reactions and immune responses in mice fed different rice cultivars including Yukihikari.

\section{Materials and methods}

\section{Animals and diets}

The following study was approved by the Hokkaido University Animal Use Committee, and animals were maintained in accordance with the Hokkaido University guidelines for the care and use of laboratory animals.

Female BALB/c mice (aged 5 weeks) were purchased from Japan SLC (Hamamatsu, Japan) and housed in standard plastic cages in a temperature-controlled $\left(23 \pm 2^{\circ} \mathrm{C}\right)$ room under a $12 \mathrm{~h}$ light-dark cycle. They were allowed free access to food and water. Mice were randomly allocated to four groups and fed a synthetic diet supplemented with uncooked and polished white rice seed powder from the four different cultivars named Yukihikari, rice $\mathrm{A}$, rice $\mathrm{B}$ and rice $\mathrm{C}$ (Table 1). Rice $A$, rice $B$ and rice $C$ are cultivars of common rice, brewery rice and waxy rice, respectively. According to the $\mathrm{N}$ content of each rice seed powder, as determined by the micro-Kjeldahl method, the protein contents were estimated as 208, 208, 212 and $202 \mathrm{~g} / \mathrm{kg}$ for Yukihikari, rice $A$, rice $B$ and rice $C$, respectively. A total of twentythree mice (Yukihikari $(n 5)$, rice $\mathrm{A}(n 6)$, rice $\mathrm{B}(n 6)$ and rice $\mathrm{C}(n 6))$ were subjected to the treatment for the induction of allergic diarrhoea, and an additional twenty-three mice (Yukihikari $(n 5)$, rice $\mathrm{A}(n 6)$, rice $\mathrm{B}(n 6)$ and rice $\mathrm{C}(n 6)$ ) were subjected to the treatment for oral immunisation as described below.

\section{Experimental design for allergic diarrhoea model}

After feeding the test diets for 3 weeks, allergic diarrhoea was induced in systemically immunised mice according to Kweon et al. ${ }^{(34)}$. Mice were immunised subcutaneously with $1 \mathrm{mg}$ OVA (grade V; Sigma, St Louis, MO, USA) in $100 \mu$ of complete Freund adjuvant (Difco Laboratories, Detroit, MI, USA). At 2 weeks after immunisation, mice were repeatedly

Table 1. Composition of the experimental diet

\begin{tabular}{lc}
\hline Ingredient & $\mathrm{g} / \mathrm{kg}$ \\
\hline Rice seed powder & $659 \cdot 5$ \\
Casein & $170 \cdot 0$ \\
Soyabean oil & $70 \cdot 0$ \\
Cellulose & $50 \cdot 0$ \\
Mineral mixture (AIN-93G-MX) & $35 \cdot 0$ \\
Vitamin mixture (AIN-93-VX) & $10 \cdot 0$ \\
L-Cystine & $3 \cdot 0$ \\
Choline bitartrate & $2 \cdot 5$
\end{tabular}

AIN, American Institute of Nutrition.

*Each of the four kinds of rice cultivars, i.e. Yukihikari, common rice (rice A), brewery rice (rice $B$ ), and waxy rice (rice $\mathrm{C}$ ), was used individually. 
given $10 \mathrm{mg}$ OVA dissolved in $100 \mu \mathrm{l}$ PBS by intragastric administration every other day. Fresh faeces were obtained before immunisation and subjected to molecular biological analyses of the microbiota as described below. Before immunisation and after the last (10th) oral administration of OVA, blood samples were obtained from the submandibular vein and subjected to ELISA for measurement of OVAspecific antibody titres as described below.

\section{Experimental design for oral sensitisation model}

After feeding the test diets for 3 weeks, mice were subjected to oral immunisation with OVA according to Yamaguchi et al. ${ }^{(35)}$. PBS $(0.2 \mathrm{ml})$ containing $0.1 \mathrm{mg}$ OVA was intragastrically administered five times per week for 11 weeks. Blood samples were obtained from the submandibular vein at weekly intervals and subjected to ELISA for measurement of OVA-specific antibody titres as described below. On the last day of the experiment, mice were anaesthetised by diethyl ether and killed by exsanguination from the carotid artery. Following a laparotomy, a $5 \mathrm{~cm}$ section of ileum and colon was excised and subjected to an in vitro permeation experiment as described below.

\section{Profile analysis of faecal microbiota by PCR-denaturing gradient gel electrophoresis}

DNA was isolated from fresh faeces using a faecal DNA isolation kit (MO Bio Laboratories, Carlsbad, CA, USA) according to the manufacturer's instructions. DNA samples were used as a template to amplify the 16S rRNA gene fragments with the universal primers U968-GC (CGC CCG GGG CGC GCC CCG GGC GGG GCG GGG GCA CGG GGG GAA CGC GAA GAA CCT TAC) and L1401 (CGG TGT GTA CAA GAC CC) ${ }^{(36)}$. Denaturing gradient gel electrophoresis (DGGE) analysis of the amplicon was performed as previously described ${ }^{(25)}$. Quantity One software (version 4.6.0; Bio-Rad, Hercules, CA, USA) was used for band identification and normalisation of band patterns from DGGE gels. Subsequently, a dendrogram of the DGGE-band profile was constructed using Pearson's curve-based correlation and the unweighted pair-group method with arithmetic mean clustering method in Quantity One software as previously described $^{(37)}$.

\section{Analysis of the 16S rRNA gene sequences in faecal bacteria}

Faecal DNA samples isolated from individual mice as described above were pooled in each group and used as a template to amplify the $16 \mathrm{~S}$ rRNA gene fragments with the universal primers U968 (AAC GCG AAG AAC CTT AC) and L1401. PCR was performed in a reaction volume of $25 \mu \mathrm{l}$ that contained $500 \mathrm{nM}$ each of primers, $1 \times \mathrm{PCR}$ buffer, $0.2 \mathrm{mM}$ each of dNTPs and $1.25 \mathrm{U}$ of Taq-HS polymerase (Takara, Ohtsu, Japan). The reaction conditions were $94^{\circ} \mathrm{C}$ for $5 \mathrm{~min}$, followed by twenty cycles of $94^{\circ} \mathrm{C}$ for $30 \mathrm{~s}, 56^{\circ} \mathrm{C}$ for $20 \mathrm{~s}$, and $68^{\circ} \mathrm{C}$ for $40 \mathrm{~s}$, and a final extension at $68^{\circ} \mathrm{C}$ for $7 \mathrm{~min}$. The amplicons were purified by a QIAquick PCR purification kit (Qiagen, Tokyo, Japan) and cloned into pGEM-Easy $\mathrm{T}$ vectors (Promega, Madison, WI, USA). Transformation was performed with competent Escherichia coli XL-1 Blue cells, and the transformants were spread on Luria-Bertani agar plates supplemented with ampicillin $(25 \mu \mathrm{g} / \mathrm{ml})$, 5-bromo-4chloro-3-indolyl- $\beta$-D-galactopyranoside $(30 \mu \mathrm{g} / \mathrm{ml})$ and isopropyl- $\beta$-D-thiogalactopyranoside $(20 \mu \mathrm{g} / \mathrm{ml})$ and incubated overnight at $37^{\circ} \mathrm{C}$. White colonies were randomly picked from each sample and grown on Luria-Bertani agar. Clones carrying inserts were identified by colony PCR using a Colony PCR M13 set (Nippongene, Tokyo, Japan). Plasmid DNA in the positive clones were amplified for sequencing with an Illustra TempliPhi DNA amplification Kit (GE Healthcare Bioscience, Tokyo, Japan) according to the manufacturer's instructions. Resultant amplicons were sequenced using an ABI3730XL or ABI3730 (Applied Biosystems, Carlsbad, CA, USA) with M13-F (GTT TTC CCA GTC ACG ACG TT) as a sequencing primer. Sequence data were aligned with the CLUSTAL W package ${ }^{(38)}$ and corrected by manual inspection. The sequences were compared with the public Ribosomal Database Project (http://rdp.cme.msu.edu/). Naive Bayesian rRNA classifier version 2.0 from Ribosomal Database Project was used to assign 16S rRNA gene sequences to the taxonomical hierarchy proposed in Bergey's Manual of Systematic Bacteriology, release $6.0^{(39)}$, with a setting threshold value of $90 \%$. Analysis of the $16 \mathrm{~S}$ rRNA gene sequences was also performed using the GenBank DNA database and the Basic Local Assignment Search Tool (BLAST) algorithm.

Real-time quantitative PCR for Akkermansia muciniphila in faeces

Abundance of Akkermansia muciniphila in faecal DNA samples isolated from individual mice as described above was determined by real-time quantitative PCR (RT-qPCR) according to Collado et al. ${ }^{(40)}$. In brief, amplification and detection of faecal DNA were performed with the Thermal Cycler Dice Real Time System (Takara). An A. muciniphila species-specific primer pair was used. RT-qPCR was performed in a reaction volume of $25 \mu$ l, containing $12.5 \mu \mathrm{l}$ SYBR Premix Ex Taq (Takara), $200 \mathrm{~nm}$ each of the forward and reverse primers (AM1, CAG CAC GTG AAG GTG GGG AC; AM2, CCT TGC GGT TGG CTT CAG AT) and $1 \mu \mathrm{l}$ of faecal DNA samples. The reaction conditions were $95^{\circ} \mathrm{C}$ for $5 \mathrm{~min}$, followed by forty cycles at $95^{\circ} \mathrm{C}$ for $15 \mathrm{~s}$, $60^{\circ} \mathrm{C}$ for $40 \mathrm{~s}$, and $72^{\circ} \mathrm{C}$ for $30 \mathrm{~s}$, and a final extension at $72^{\circ} \mathrm{C}$ for $5 \mathrm{~min}$. Collado et al. ${ }^{(40)}$ reported that the primer pair is specific for A. muciniphila at $60^{\circ} \mathrm{C}$. The fluorescent products were detected at the last step of each cycle. A melting curve analysis was made after amplification to distinguish the targeted PCR product from the non-targeted PCR product. All samples were analysed in duplicate.

Using a pooled faecal DNA sample from mice fed rice A as a template, a fragment of $16 \mathrm{~S}$ rDNA was amplified by PCR with the A. muciniphila species-specific primer pair (AM1 and AM2 as described above). The size of the amplicon, estimated by agarose gel electrophoresis, was identical to the expected size $(327 \mathrm{bp})^{(40)}$, and the sequence was completely identical to A. muciniphila (data not shown). The amplicon was purified and subcloned into a bacterial plasmid as described above. The plasmid DNA was extracted with the QIAprep Spin Miniprep kit (Qiagen) and used as a standard for RT-qPCR. 


\section{ELISA for antibody measurements}

The serum titres of antibodies specific to OVA were determined by ELISA as previously described ${ }^{(35)}$. In brief, IgG, IgG1 and IgG2a were captured with OVA-coated wells and detected with horseradish peroxidase (HRP)-conjugated goat anti-mouse IgG polyclonal antibody (Zymed Laboratories, South San Francisco, CA, USA), rat anti-mouse IgG1 monoclonal antibody (mAb) (clone LO-MG1-2; Zymed Laboratories) and rat anti-mouse $\operatorname{IgG} 2 \mathrm{a} \mathrm{mAb}$ (clone LO-MG2a-3; Zymed Laboratories), respectively. IgE was captured with rat anti-mouse IgE mAb (LO-ME-2; Zymed Laboratories) and detected with digoxigenin (DIG)-conjugated OVA followed by HRP-conjugated sheep anti-DIG Fab fragments (Roche Diagnostics, Tokyo, Japan). Pre-immunised serum was used as a negative control. The average extinction in negative control wells, to which three times the standard deviation was added, provided the reference for determination of the titre in the test sera. Antibody titres were expressed as the reciprocal of the last dilution yielding an extinction value higher than the reference value.

\section{Permeation of horseradish peroxidase in the ileum and colon} in vitro

Gut permeability was measured using translocation of HRP in isolated segments of ileum and colon, according to Enomoto et al. ${ }^{(41)}$. Briefly, $5 \mathrm{~cm}$ segments of ileum and colon were everted, filled with $200 \mu \mathrm{l}$ Tris buffer $(125 \mathrm{~mm}-\mathrm{NaCl}$, $10 \mathrm{~mm}$-fructose, $30 \mathrm{~mm}$-2-amino-2-hydroxymethyl-propane1,3-diol; $\mathrm{pH} 7 \cdot 5$ ), and ligated at both ends. The filled gut segments were incubated in Tris buffer containing HRP $(40 \mu \mathrm{g} / \mathrm{ml})$ (Sigma) at $37^{\circ} \mathrm{C}$. After $30 \mathrm{~min}$, gut sacs were removed and the contents of each sac were collected. HRP activity in the contents of each sac was determined spectrophotometrically from the rate of oxidation of $3,3^{\prime}, 5,5^{\prime}$-tetramethylbenzidine (Sigma).

\section{Statistical analysis}

Results are presented as mean values with their standard errors or as individual values for each mouse. We assessed statistical significance using a $P$ value calculated with the KruskalWallis non-parametric test, while the $\chi^{2}$ test was used to compare the frequencies of diarrhoea. Data analysis was performed with StatView for Macintosh (version 5.0; SAS Institute, Inc., Cary, NC, USA). $P<0.05$ was considered statistically significant.

\section{Results}

Comparison of faecal microbiota in mice fed different rice cultivars

The 16S rRNA gene profiles of the bacterial collections in the faecal samples were generated by PCR coupled with DGGE. Fig. 1 shows the DGGE band profile of all mice subjected to the experiment. The intensity and position of detected bands were subjected to cluster analysis. The dendrogram shows two large clusters of mice fed rice A and those fed the other three cultivars (Fig. 1(B)). In the latter cluster, mice fed Yukihikari constituted a sub-cluster. Thus, it appears that the gut microbiota composition was different between mice fed Yukihikari and those fed rice A. To further investigate the difference, 16S rRNA clone libraries were constructed from DNA samples pooled in each dietary group, and eightyfour and seventy-seven sequences were analysed for the Yukihikari group and rice A group, respectively (Table 2). The phylum Firmicutes was the most abundant taxonomic group in both the Yukihikari and rice A groups (60.9 and $41.6 \%$, respectively). In the Yukihikari group, the phylum Proteobactera was the second most predominant group (29.5\%). Although the phylum Verrucomicrobia was the second most abundant group in the rice A group (24.6\%), the Yukihikari group contained small numbers of bacteria

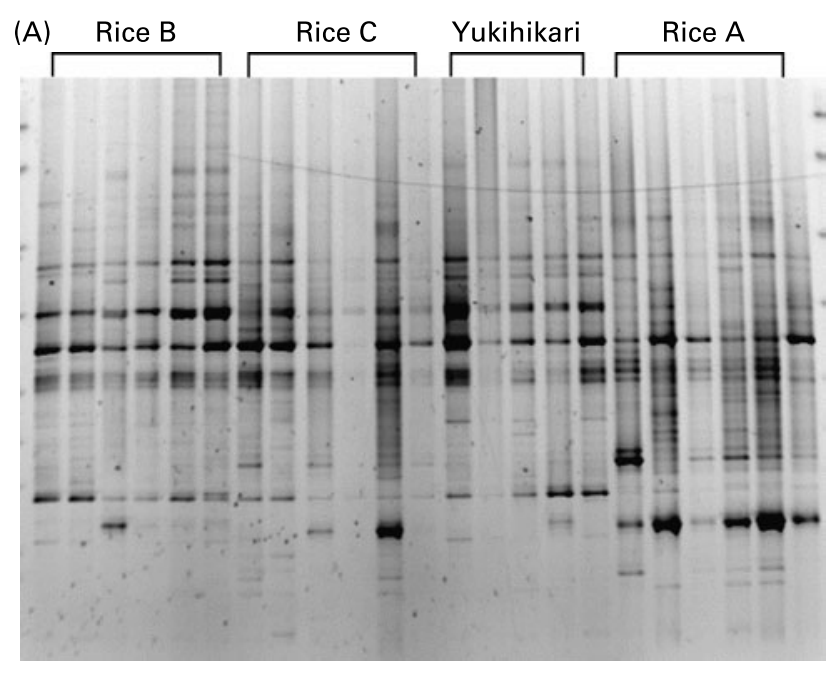

(B)

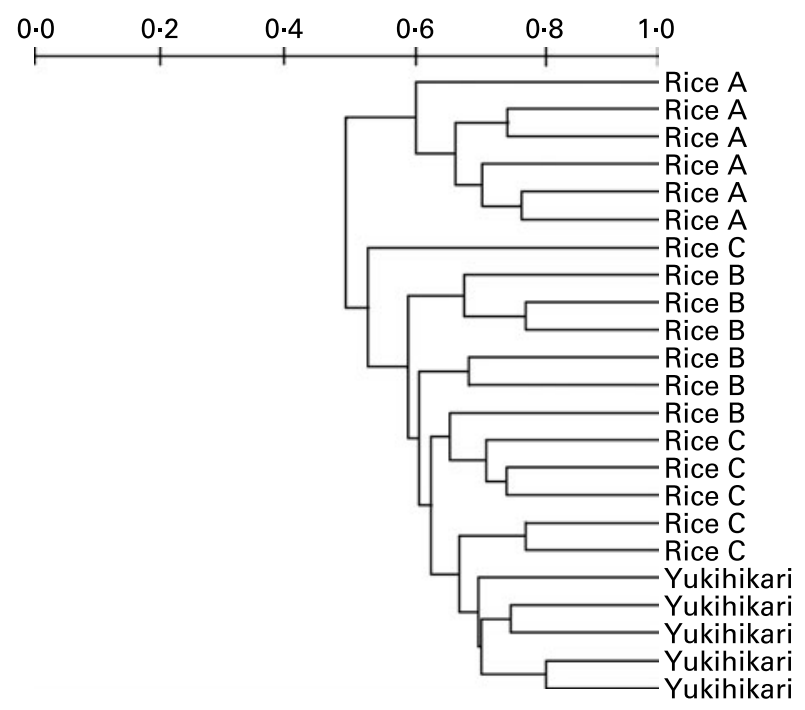

Fig. 1. PCR-denaturing gradient gel electrophoresis (DGGE) analysis of faecal microbiota based on 16S rRNA gene sequences in BALB/c mice fed different rice cultivars: common rice (rice A); brewery rice (rice B); waxy rice (rice C); Yukihikari rice. (A) SYBR green staining of PCR products separated by DGGE. (B) Similarities among DGGE band profiles of faecal bacteria of mice were calculated based on the position and intensity of bands, and the dendrogram of DGGE band profiles was constructed by the unweighted pair-group method with arithmetic mean (UPGMA) clustering method. Distances are measured in arbitrary units. 
Table 2. Taxonomic distribution of $16 \mathrm{~S}$ rRNA gene sequences in faeces of $B A L B / c$ mice fed different cultivars of rice

\begin{tabular}{|c|c|c|c|c|}
\hline \multirow[t]{2}{*}{ Sequences... } & \multicolumn{2}{|c|}{ Yukihikari } & \multicolumn{2}{|c|}{ Rice A } \\
\hline & $n$ & $\%$ & $n$ & $\%$ \\
\hline Phylum Actinobacteria & 2 & 2.4 & 3 & 3.9 \\
\hline Class Actinobacteria & 2 & $2 \cdot 4$ & 3 & 3.9 \\
\hline Phylum Bacteroidetes & 3 & 3.6 & 11 & 14.3 \\
\hline Class Bacteroidetes & 3 & 3.6 & 11 & 14.3 \\
\hline Phylum Firmicutes & 51 & $60 \cdot 9$ & 32 & 41.6 \\
\hline Class Bacilli & 2 & 2.4 & 12 & $15 \cdot 6$ \\
\hline Class Clostridia & 45 & 53.7 & 12 & $15 \cdot 6$ \\
\hline Unclassified Firmicutes & 4 & 4.8 & 8 & $10 \cdot 4$ \\
\hline Phylum Proteobacteria & 25 & 29.5 & 8 & $10 \cdot 4$ \\
\hline Class Deltaproteobacteria & 20 & 23.9 & 5 & 6.5 \\
\hline Class Epsilonproteobacteria & 5 & 5.6 & 3 & 3.9 \\
\hline Phylum Verrucomicrobia & 2 & $2 \cdot 4$ & 19 & 24.6 \\
\hline Class Verrucomicrobiae & 2 & $2 \cdot 4$ & 19 & 24.6 \\
\hline Unclassified bacteria & 1 & $1 \cdot 2$ & 4 & $5 \cdot 2$ \\
\hline Total & 84 & $100 \cdot 0$ & 77 & $100 \cdot 0$ \\
\hline
\end{tabular}

Rice A, common rice.

belonging to the phylum Verrucomicrobia (2.4\%). Because all sequences in the phylum Verrucomicrobia were classified to the genus Akkermansia (similarity $100 \%$ ), RT-qPCR with a species-specific primer pair was used to estimate the abundance of A. muciniphila in the faecal samples of each mouse (Fig. 2). Although there was no significant difference among the groups, the levels tended to be lower in mice fed Yukihikari than those fed rice $\mathrm{A}$, rice $\mathrm{B}$ and rice $\mathrm{C}$.

\section{Comparison of allergic diarrhoea in mice fed different rice cultivars}

Fig. 3 shows the time course of changes in the number of mice affected with diarrhoea. The total number of mice subjected to the experiment was five, six, six and six in the Yukihikari, rice

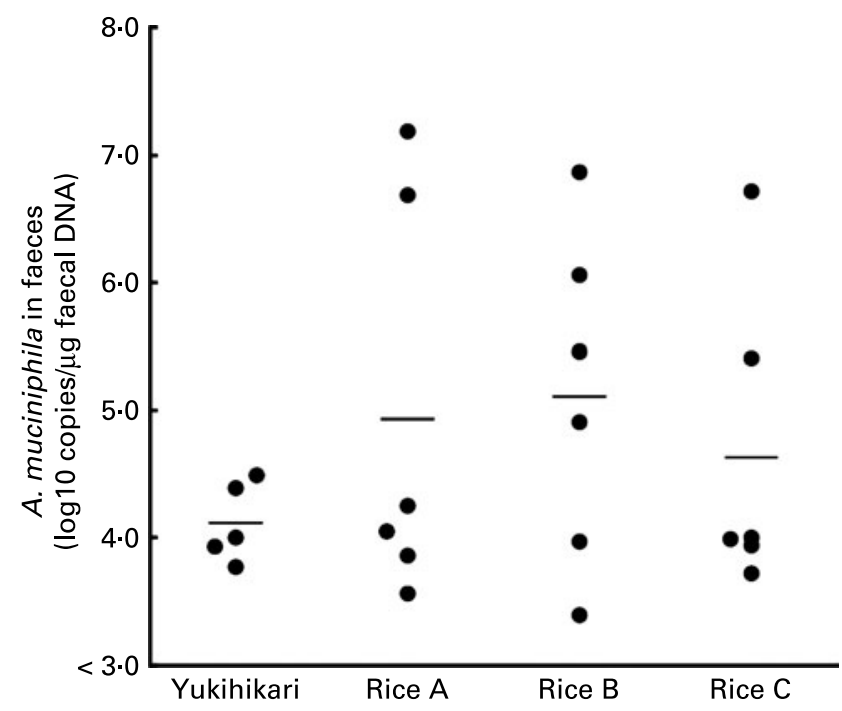

Fig. 2. Abundance of Akkermansia muciniphila in faeces of BALB/c mice fed different rice cultivars: common rice (rice $A$ ); brewery rice (rice $B$ ); waxy rice (rice $\mathrm{C}$ ); Yukihikari rice. Real-time quantitative PCR with a species-specific primer pair was performed to estimate the abundance of $A$. muciniphila. (๑), Values of individual mice; $(-)$, mean values.

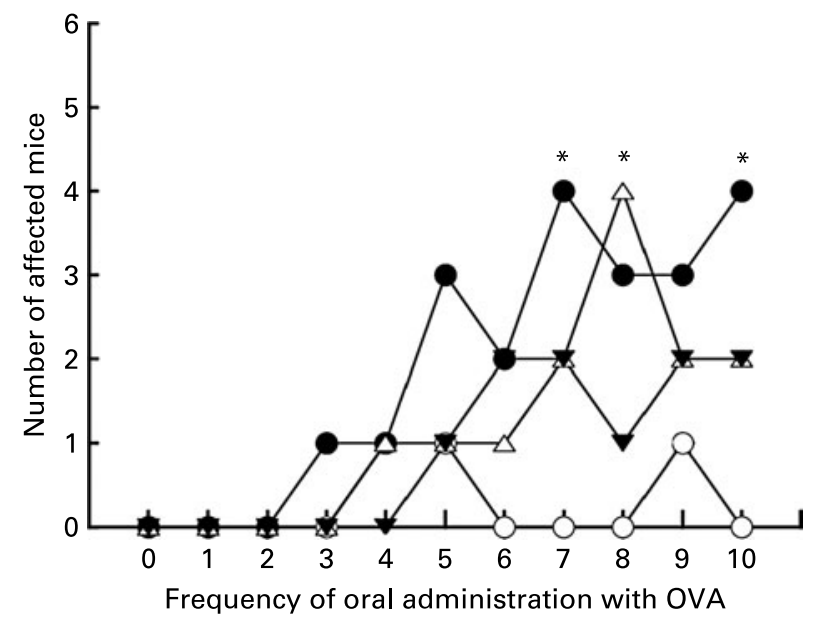

Fig. 3. Changes in time course of the number of animals affected with ovalbumin (OVA)-induced diarrhoea in sensitised BALB/c mice fed different rice cultivars: common rice (rice $A ;-\bullet-$ ); brewery rice (rice $B ;-\triangle-$ ); waxy rice (rice $\mathrm{C} ;-\mathbf{\nabla}-$ ); Yukihikari rice $\left(-\mathrm{O}_{-}\right)$. The total numbers of mice subjected to the experiment were: Yukihikari, $n 5$; rice $\mathrm{A}, n 6$; rice $\mathrm{B}, n 6$; rice $\mathrm{C}, n 6$. ${ }^{*}$ Value was significantly different from that in the Yukihikari group ( $\chi^{2}$ test).

$A$, rice $B$ and rice $C$ groups, respectively. Diarrhoea was observed after three to five oral administrations of OVA to the systemically immunised mice. Repeated oral administration of OVA in mice without systemic immunisation did not induce any change in the gross appearance of faeces (data not shown). As described by Kweon et al. ${ }^{(34)}$, diarrhoea was observed within $30 \mathrm{~min}$ after oral OVA administration and the effect decayed within $2 \mathrm{~h}$, suggesting that an acute allergic response had occurred in these mice. In mice fed rice $\mathrm{A}$, rice $\mathrm{B}$ and rice $\mathrm{C}$, the incidence of diarrhoea roughly continued to increase by the end of the experiment, whereas mice fed Yukihikari showed a lower incidence of diarrhoea throughout the induction period. In particular, mice fed rice A showed a significantly higher incidence of diarrhoea as compared with mice fed Yukihikari after the 7th and 10th oral administration of OVA. Likewise, the incidence after the 8th administration was significantly higher in mice fed rice B than in those fed Yukihikari.

Fig. 4 shows serum antibody titres specific to OVA before starting oral OVA administration and after the last administration. In both IgG (Fig. 4(A)) and IgE (Fig. 4(B)), the titres tended to be increased by oral administration of OVA. However, there was no significant difference in both IgG and $\operatorname{IgE}$ titres among the groups. Mice without systemic immunisation showed undetectable levels of both $\mathrm{IgG}$ and IgE antibodies specific to OVA (data not shown).

\section{Comparison of oral sensitisation in mice fed different rice cultivars}

Fig. 5(A) shows the time course of changes in the titres of serum $\operatorname{IgG}$ antibodies against orally administered OVA. In mice fed rice $A$ and rice $C$, the anti-OVA $\operatorname{IgG}$ titres began increasing at 2 weeks after starting administration of OVA and stabilised at 7-8 weeks. The anti-OVA IgG titres tended to be lower in mice fed Yukihikari than in mice fed rice $\mathrm{A}$ and rice $\mathrm{C}$ throughout the experimental period; levels 

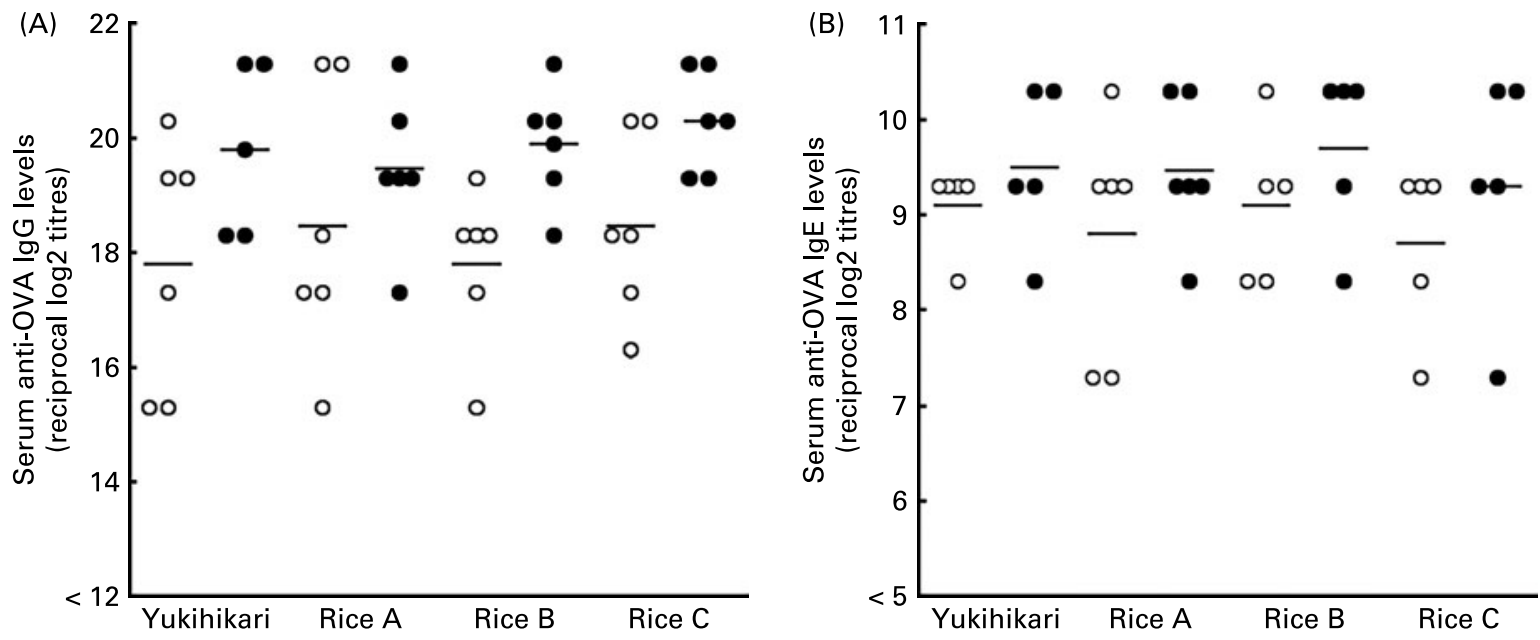

Fig. 4. Serum IgG (A) and IgE (B) antibody titres specific to ovalbumin (OVA) in BALB/c mice fed different rice cultivars (common rice (rice A); brewery rice (rice B); waxy rice (rice C); Yukihikari rice) in the allergic diarrhoea experiment. $(\bigcirc)$, Values of individual mice before immunisation; $(\bullet)$, values of individual mice after the last oral administration of OVA; $(-)$, mean values.

in mice fed rice B were intermediate. In particular, there was a significant difference between mice fed Yukihikari and those fed rice $\mathrm{C}$ at 6 weeks after starting OVA administration. Additionally, the levels were significantly lower in mice fed
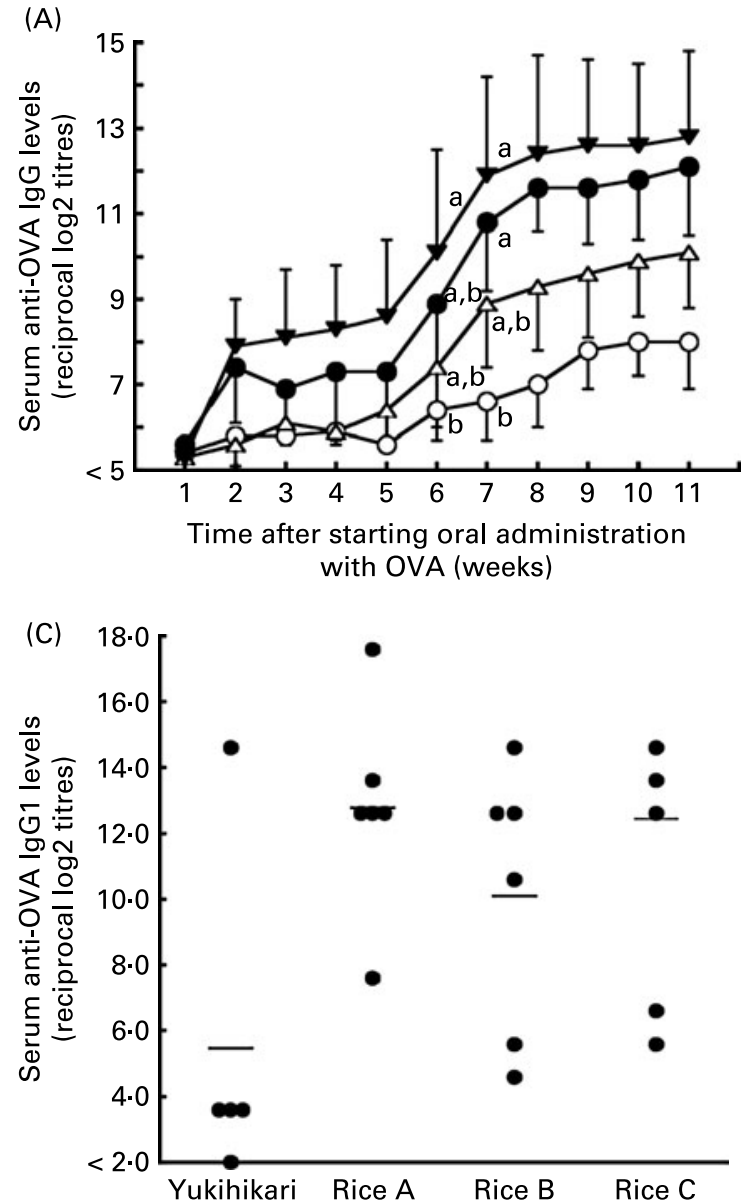

Yukihikari than in those fed rice $\mathrm{C}$ and rice $\mathrm{A}$ at 7 weeks after starting OVA administration. After the last oral administration of OVA, the anti-OVA IgE titres tended to be lower in mice fed Yukihikari and rice $B$ than in those fed rice $A$ and
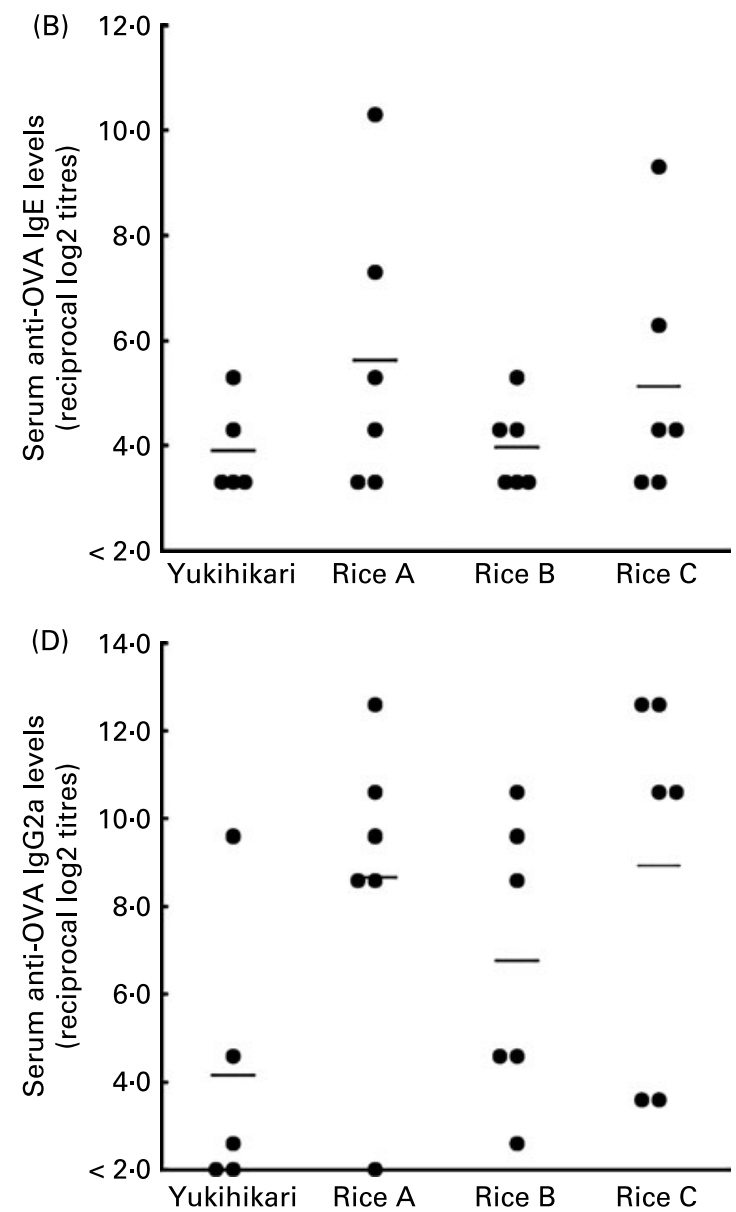

Fig. 5. (A) Changes in time course of serum antibody titres specific to ovalbumin (OVA) in BALB/c mice fed different rice cultivars (rice A; - -); brewery rice (rice $\mathrm{B} ;-\triangle-$ ); waxy rice (rice $\mathrm{C} ;-\mathbf{\nabla}-$ ); Yukihikari rice $\left(-\mathrm{O}_{-}\right.$) in the oral sensitisation experiment. Values are means, with standard errors represented by vertical bars. ${ }^{a, b}$ Mean values with unlike letters were significantly different $(P<0.05)$. (B) Serum IgE, (C) serum IgG1 and (D) serum IgG2a titres specific to OVA. ( $\bullet$, Values of individual mice; $(-)$, mean values. 


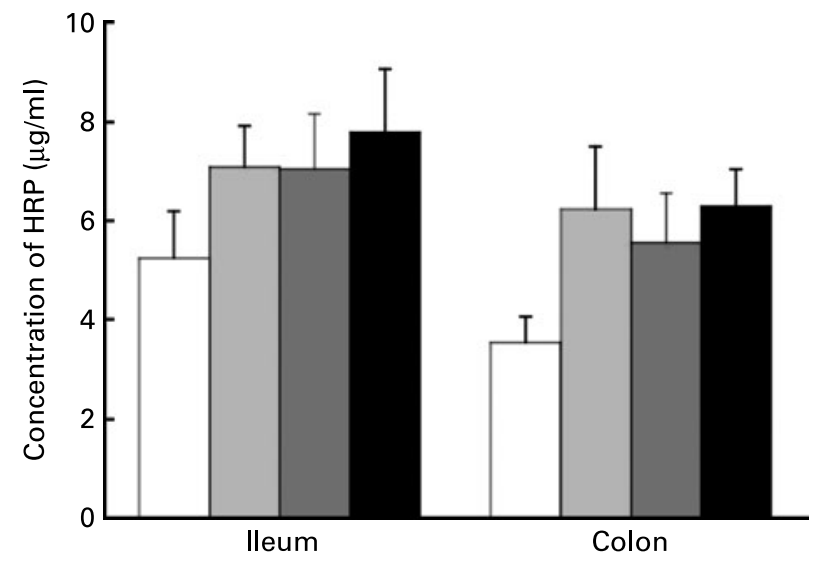

Fig. 6. In vitro gut permeability in BALB/c mice fed different rice cultivars: common rice (rice $A ; \square$ ); brewery rice (rice $B$; $\square$ ); waxy rice (rice $C ; \mathbf{\square}$ ); Yukihikari rice $(\square)$. Gut permeability was estimated using translocation of horseradish peroxidase (HRP) in isolated segments of ileum and colon. Values are means, with standard errors represented by vertical bars.

rice $\mathrm{C}$ (Fig. 5(B)). Similar to the OVA-IgG titres (Fig. 5(A)), the OVA-IgG1 and OVA-IgG2a titres tended to be lower in mice fed Yukihikari than in those fed the other three rice cultivars (Fig. 5(C) and (D), respectively).

\section{Comparison of in vitro gut permeability in mice fed different rice cultivars}

Gut permeability was estimated using translocation of HRP in isolated segments of ileum and colon (Fig. 6). Although there were no significant differences among the groups, the translocation of HRP tended to be lower in mice fed Yukihikari than in those fed rice $A$, rice $B$ and rice $C$, in both the ileum and colon.

\section{Discussion}

Our small-scale prospective open clinical trial showed that consumption of Yukihikari, a cultivar of common rice developed in Japan, improved the clinical skin score of AD associated with a suspected rice allergy ( $\mathrm{T}$ Yanagihara, unpublished results). Concerning this preliminary observation, we postulated that resistant starch is involved in the ameliorating effect of Yukihikari on AD by modulating gut microbiota and/or by potentiating intestinal barrier function.

Amylose contents in the rice seed powder of Yukihikari, rice $\mathrm{A}$, rice $\mathrm{B}$ and rice $\mathrm{C}$ were $18,18,20$ and $0 \%$, respectively ( $\mathrm{T}$ Yanagihara, unpublished results). Additionally, the resistant starch contents, as determined by in vitro enzymic digestion, in the rice seed powders were $1.9,0.9,1.7$ and $1.2 \mathrm{mg} / \mathrm{g}$ for Yukihikari, rice $\mathrm{A}$, rice $\mathrm{B}$ and rice $\mathrm{C}$, respectively (T Ogasawara, T Yanagihara, Y Tokunaga and K Sonoyama, unpublished results). In the present study, therefore, the resistant starch content was not necessarily correlated with amylose content in the rice powders, and it appears that mice consumed extremely small amounts of resistant starch in each diet. Nevertheless, 16S rRNA-based analyses clearly demonstrated that the composition of faecal microbiota was different in mice fed different rice cultivars, particularly between mice fed Yukihikari and those fed rice A. The results suggest that consumption of different rice cultivars modulates the gut microbiota in mice, even though this effect might not be attributed to resistant starch. Therefore, it remains to be elucidated what rice constituents modulate the gut microbiota. Additionally, we should be cautious in extrapolating such mice data to humans because of difference in the composition of gut microbiota between mice and humans. Furthermore, mice were fed the uncooked rice seed powder in the present study, while humans usually consume cooked rice which naturally contains smaller amounts of resistant starch as compared with uncooked rice. Therefore, further studies are needed to clarify the relationship between rice consumption and gut microbiota.

Although the present study showed that the phylum Firmicutes and the phylum Proteobacteria in faeces were more abundant in mice fed Yukihikari than in mice fed rice A, it remains unclear whether these changes are related to allergic reactions and/or immune responses. However, it is noteworthy that the abundance of $A$. muciniphila tended to be lower in faeces of mice fed Yukihikari than in those fed the other three cultivars, because A. muciniphila is a mucin-degrading bacterium in the intestine ${ }^{(42)}$. Mucins are high-molecular mass glycoproteins and the main constituents of the mucus covering the epithelial surface of the gastrointestinal tract, and thus contribute to the epithelial protective barrier against pathogenic micro-organisms, as well as chemical, physical or enzymic damage. Therefore, degradation of intestinal mucins possibly results in reduced barrier function, which in turn may cause increased uptake of allergenic proteins in the gastrointestinal tract. The present study showed that the translocation of HRP in isolated segments of ileum and colon tended to be lower in mice fed Yukihikari than in those fed the other three cultivars, suggesting that lower abundance of A. muciniphila in the gut of mice fed Yukihikari are accompanied by higher integrity of intestinal barrier function and lower intestinal permeation of food antigens.

Additionally, the present study initially assumed that resistant starch in Yukihikari potentiates intestinal barrier function, because Toden et al. ${ }^{(31,32)}$ and our previous study ${ }^{(29,30)}$ showed that consumption of resistant starch potentiates intestinal barrier function as a result of an increased mucus layer in rats. However, the resistant starch contents in the rice seed powders in the present study (approximately $0 \cdot 1-0.2 \%$ ) were much lower as compared with those in high-amylose maize starch used in Toden et al. ${ }^{(31,32)}$ and our previous study ${ }^{(29,30)}$ (approximately 30-40\%). Therefore, lower intestinal permeability in mice fed Yukihikari may be attributed to not resistant starch but rather lower abundance of A. muciniphila.

Given that the gut microbiota influences normal development of the immune system, and in turn affects the development of allergic diseases ${ }^{(13,14)}$, the ameliorating effect of Yukihikari on $\mathrm{AD}$ might be not limited to rice allergy. Indeed, the present study showed that the incidence of OVA-induced allergic diarrhoea was lower in mice fed Yukihikari than in those fed rice $\mathrm{A}$, rice $\mathrm{B}$ and rice $\mathrm{C}$. The results suggest that consumption of Yukihikari protects against acute gut allergy in mice. In these mice, however, the serum antibody titres specific to OVA were the same among the groups, suggesting that gut microbiota modulation by consuming Yukihikari has no impact on the systemic sensitisation 
with OVA. Additionally, the present study revealed that the increase in serum antibody titres specific to orally administered OVA was lower in mice fed Yukihikari than in those fed the other three cultivars. The data suggest that consumption of Yukihikari protects against oral sensitisation in mice. In our preliminary experiments, however, there were no differences in contact hypersensitivity induced by topical application of 2,4-dinitorofluorobenzene in BALB/c and C57BL/6 mice fed the four cultivars (K Sonoyama, $\mathrm{S}$ Honma, $\mathrm{T}$ Ogasawara, $\mathrm{N}$ Sasajima, $\mathrm{Y}$ Tokunaga and $\mathrm{T}$ Yanagihara, unpublished results). These findings suggest that consumption of Yukihikari might affect the intestinal uptake of orally administered antigen, but not systemic immune responses. Thus, it is possible that a lower incidence of OVA-induced allergic diarrhoea and lower antibody responses against orally administered OVA are attributed to higher integrity of intestinal barrier function, which is associated with a lower abundance of $A$. muciniphila. In the present study, however, we observed no significant correlation between the faecal excretions of A. muciniphila and the frequency of allergic diarrhoea in individual mice (data not shown). Therefore, further studies are needed to clarify the relationship between A. muciniphila in the gut and allergic reactions.

Alternatively, the reduction of antibody responses against orally administered OVA in mice fed Yukihikari might be attributed, in part, to the induction of oral tolerance. Oral tolerance is characterised by a state of systemic immune non-responsiveness towards antigens present in the gastrointestinal tract ${ }^{(43)}$, and this mechanism presumably prevents the development of food allergy. As several studies have reported that the gut microbiota plays a crucial role in the induction of oral tolerance $\mathrm{e}^{(44-46)}$, it is likely that the gut microbiota in mice fed Yukihikari might be advantageous in the induction of tolerance against orally administered OVA. Therefore, we are now conducting experiments that investigate the effect of different rice cultivars on the induction of oral tolerance in mice.

Taken together, the present study indicated that the composition of gut microbiota was modulated by the consumption of different rice cultivars, and that mice fed Yukihikari showed a lower incidence of OVA-induced allergic diarrhoea and lower antibody responses against orally administered OVA in mice. These results suggest that the gut microbiota in mice fed Yukihikari is beneficial in the prevention of food allergy. In particular, it is interesting to note that lower abundance of the mucin-degrader A. muciniphila in the gastrointestinal tract is associated with the consumption of Yukihikari in mice. In addition to rigorously controlled clinical trials, however, mechanistic studies are required to further elucidate the allergy-preventing and/or -ameliorating effect of Yukihikari.

\section{Acknowledgements}

The present study was partly supported by Special Coordination Funds for Promoting Science and Technology, by a Grant-in-Aid for Scientific Research from The Ministry of Education, Science, Sports and Culture of Japan, and by The Iijima Memorial Foundation.
K. S. and T. Yanagihara were involved in designing the study. K. S. wrote the manuscript. T. O., H. I. and T. M. were involved in the animal experiments. H. G. and T. Yoshida performed the in vitro permeation experiment. N. T., R. F. and J. W. performed the molecular biological analyses of gut microbiota. Y. T. was involved in the preparation of rice seed powder.

None of the authors has a conflict of interest.

\section{References}

1. Ikezawa Z, Miyakawa K, Komatsu H, et al. (1992) A probable involvement of rice allergy in severe type of atopic dermatitis in Japan. Acta Derm Venereol Suppl (Stockh) 176, 103-107.

2. Miyakawa K, Hirai Y, Miyakawa J, et al. (1988) Statistical analyses of the diagnostic criteria, clinical severity, IgE-RAST score, and serum IgE value in patients with atopic dermatitis (AD) - probable involvement of food antigens, especially rice, in severe cases (article in Japanese). Arerugi 37, 1101-1110.

3. Yamada K, Kishimoto M, Inagaki Y, et al. (1985) Investigation on food allergens in atopic dermatitis (article in Japanese). Shonika Rinsho 38, 2545-2560.

4. Shibasaki M, Suzuki S, Nemoto H, et al. (1979) Allergenicity and lymphocyte-stimulating property of rice protein. J Allergy Clin Immunol 64, 259-265.

5. Urisu A, Yamada K, Masuda S, et al. (1991) 16-Kilodalton rice protein is one of the major allergens in rice grain extract and responsible for cross-allergenicity between cereal grains in the Poaceae family. Int Arch Allergy Appl Immunol 96, 244-252.

6. Izumi H, Adachi T, Fujii N, et al. (1992) Nucleotide sequence of a cDNA clone encoding a major allergenic protein in rice seeds. Homology of the deduced amino acid sequence with members of $\alpha$-amylase/trypsin inhibitor family. FEBS Lett 302, 213-216.

7. Nakase M, Adachi T, Urisu A, et al. (1996) Rice (Oryza sativa L.) $\alpha$-amylase inhibitors of $14-16 \mathrm{kDa}$ are potential allergens and products of a multigene family. J Agric Food Chem 44, 2624-2628.

8. Limas GG, Salinas M, Moneo I, et al. (1990) Purification and characterization of ten new rice NaCl-soluble proteins: identification of four protein-synthesis inhibitors and two immunoglobulin-binding proteins. Planta 181, 1-9.

9. Usui Y, Nakase M, Hotta H, et al. (2001) A 33-kDa allergen from rice (Oryza sativa L. Japonica). cDNA cloning, expression, and identification as a novel glyoxalase I. J Biol Chem 276, 11376-11381.

10. Watanabe M, Miyakawa M, Ikezawa Z, et al. (1990) Production of hypoallergenic rice by enzymatic decomposition of constituent proteins. J Food Sci 55, 781-783.

11. Kato T, Katayama E, Matsubara S, et al. (2000) Release of allergenic proteins from rice grains induced by high hydrostatic pressure. J Agric Food Chem 48, 3124-3129.

12. Tada Y, Nakase M, Adachi T, et al. (1996) Reduction of $14-16 \mathrm{kDa}$ allergenic proteins in transgenic rice plants by antisense gene. FEBS Lett 391, 341-345.

13. Wills-Karp M, Santeliz J \& Karp CL (2001) The germless theory of allergic disease: revisiting the hygiene hypothesis. Nat Rev Immunol 1, 69-75.

14. Macpherson AJ \& Harris NL (2004) Interactions between commensal intestinal bacteria and the immune system. Nat Rev Immunol 4, 478-485.

15. Kalliomaki M, Salminen S, Arvilommi H, et al. (2001) Probiotics in primary prevention of atopic disease: a randomised placebo-controlled trial. Lancet 357, 1076-1079. 
16. Isolauri E, Arvola T, Sutas Y, et al. (2000) Probiotics in the management of atopic eczema. Clin Exp Allergy 30, $1604-1610$.

17. Gibson GR, Beatty ER, Wang X, et al. (1995) Selective stimulation of bifidobacteria in the human colon by oligofructose and inulin. Gastroenterology 108, 975-982.

18. Blaut M (2002) Relationship of prebiotics and food to intestinal microflora. Eur J Nutr 41, 11-16.

19. Sartor RB (2004) Therapeutic manipulation of the enteric microflora in inflammatory bowel diseases: antibiotics, probiotics, and prebiotics. Gastroenterology 126, 620-633.

20. Macfarlane S, Macfarlane GT \& Cummings JH (2006) Review Article: prebiotics in the gastrointestinal tract. Aliment Pharmacol Ther 24, 701-714.

21. Moro G, Arslanoglu S, Stahl B, et al. (2006) A mixture of prebiotic oligosaccharides reduces the incidence of atopic dermatitis during the first six months of age. Arch Dis Child 91, 814-819.

22. Arslanoglu S, Moro GE, Schmitt J, et al. (2008) Early dietary intervention with a mixture of prebiotic oligosaccharides reduces the incidence of allergic manifestations and infections during the first two years of life. J Nutr 138, 1091-1095.

23. Watanabe H, Sonoyama K, Watanabe J, et al. (2004) Reduction of allergic airway eosinophilia by dietary raffinose in Brown Norway rats. Br J Nutr 92, 247-255.

24. Sonoyama K, Watanabe H, Watanabe J, et al. (2005) Allergic airway eosinophilia is suppressed in ovalbumin-sensitized Brown Norway rats fed raffinose and $\alpha$-linked galactooligosaccharide. J Nutr 135, 538-543.

25. Watanabe J, Sasajima N, Aramaki A, et al. (2008) Consumption of fructo-oligosaccharide reduces 2,4-dinitrofluorobenzeneinduced contact hypersensitivity in mice. Br J Nutr $\mathbf{1 0 0}$, 339-346.

26. Fujitani S, Ueno K, Kamiya T, et al. (2007) Increased number of CCR4-positive cells in the duodenum of ovalbumin-induced food allergy model $\mathrm{Nc} / \mathrm{jic}$ mice and antiallergic activity of fructooligosaccharides. Allergol Int 56, 131-138.

27. Vos AP, van Esch BC, Stahl B, et al. (2007) Dietary supplementation with specific oligosaccharide mixtures decreases parameters of allergic asthma in mice. Int Immunopharmacol 7, 1582-1587.

28. Morita T, Ito Y, Brown IL, et al. (2007) In vitro and in vivo digestibility of native maize starch granules varying in amylose contents. J AOAC Int 90, 1628-1634.

29. Morita T, Tanabe H, Takahashi K, et al. (2004) Ingestion of resistant starch protects endotoxin influx from the intestinal tract and reduces D-galactosamine-induced liver injury in rats. J Gastroenterol Hepatol 19, 303-313.

30. Morita T, Tanabe H, Sugiyama K, et al. (2004) Dietary resistant starch alters the characteristics of colonic mucosa and exerts a protective effect on trinitrobenzene sulfonic acidinduced colitis in rats. Biosci Biotechnol Biochem 68 , 2155-2164.

31. Toden S, Bird AR, Topping DL, et al. (2005) Resistant starch attenuates colonic DNA damage induced by higher dietary protein in rats. Nutr Cancer 51, 45-51.
32. Toden S, Bird AR, Topping DL, et al. (2007) Dose-dependent reduction of dietary protein-induced colonocyte DNA damage by resistant starch in rats correlates more highly with caecal butyrate than with other short chain fatty acids. Cancer Biol Ther 6, 253-258.

33. Kalliomäki M, Salminen S \& Isolauri E (2008) Positive interactions with the microbiota: probiotics. Adv Exp Med Biol 635, 57-66.

34. Kweon MN, Yamamoto M, Kajiki M, et al. (2000) Systemically derived large intestinal $\mathrm{CD} 4^{+} \mathrm{Th} 2$ cells play a central role in STAT6-mediated allergic diarrhea. J Clin Invest 106, 199-206.

35. Yamaguchi N, Sugita R, Miki A, et al. (2006) Gastrointestinal Candida colonisation promotes sensitisation against food antigens by affecting the mucosal barrier in mice. Gut $\mathbf{5 5}$, 954-960.

36. Zoetendal EG, Akkermans AD \& de Vos WM (1998) Temperature gradient gel electrophoresis analysis of 16S rRNA from human fecal samples reveals stable and host-specific communities of active bacteria. Appl Environ Microbiol 64, 3854-3859.

37. Wasaki J, Rothe A, Kania A, et al. (2005) Root exudation, phosphorus acquisition, and microbial diversity in the rhizosphere of white lupine as affected by phosphorus supply and atmospheric carbon dioxide concentration. J Environ Qual 34, 2157-2166.

38. Thompson JD, Higgins DG \& Gibson TJ (1994) CLUSTAL W: improving the sensitivity of progressive multiple sequence alignment through sequence weighting, position-specific gap penalties and weight matrix choice. Nucleic Acid Res 22, $4673-4680$.

39. Cole JR, Chai B, Farris RJ, et al. (2005) The Ribosomal Database Project (RDP-II): sequences and tools for highthroughput rRNA analysis. Nucleic Acids Res 33 (database issue), D294-D296.

40. Collado MC, Derrien M, Isolauri E, et al. (2007) Intestinal integrity and Akkermansia muciniphila, a mucin-degrading member of the intestinal microbiota present in infants, adults, and the elderly. Appl Environ Microbiol 73, 7767-7770.

41. Enomoto N, Yamashina S, Schemmer P, et al. (1999) Estriol sensitizes rat Kupffer cells via gut-derived endotoxin. Am $J$ Physiol 277, G671-G677.

42. Derrien M, Vaughan EE, Plugge CM, et al. (2004) Akkermansia muciniphila gen. nov., sp. nov., a human intestinal mucindegrading bacterium. Int J Syst Evol Microbiol 54, 1469-1476.

43. Strobel S \& Mowat AM (1998) Immune responses to dietary antigens: oral tolerance. Immunol Today 19, 173-181.

44. Kiyono H, McGhee JR, Wannemuehler MJ, et al. (1982) Lack of oral tolerance in $\mathrm{C} 3 \mathrm{H} / \mathrm{HeJ}$ mice. J Exp Med 155, 605-610.

45. Moreau MC \& Corthier G (1988) Effect of the gastrointestinal microflora on induction and maintenance of oral tolerance to ovalbumin in $\mathrm{C} 3 \mathrm{H} / \mathrm{HeJ}$ mice. Infect Immun 56, 2766-2768.

46. Sudo N, Sawamura S, Tanaka K, et al. (1997) The requirement of intestinal bacterial flora for the development of an IgE production system fully susceptible to oral tolerance induction. J Immunol 159, 1739-1745. 